\title{
INFRARED DUST EMISSION IN GALAXIES AND SPECTRAL DISTORTION OF MICROWAVE BACKGROUND
}

\author{
V. K. Khersonskii \\ Leningrad Department of \\ the Special Astrophysical Observatory \\ USSR Academy of Sciences, Leningrad \\ Pulkovo, USSR
}

\author{
N. V. Voshchinnikov \\ Astronomical Department \\ Leningrad State University \\ Leningrad, Petrodvoretz, USSR
}

Recent observations (Matsumoto et al., 1988) indicate that at submillimeter wavelengths the spectrum of the cosmic background radiation (CBR) deviates from that of Planckian blackbody with a temperature $T_{R}^{0}=2.76 \mathrm{~K}$. The relative excess of the flux $\zeta(v)=\left[F(v)-F_{0}(v)\right] / F_{0}(v)$ (where $F(v)$ and $F_{0}(v)$ are the registered flux and the flux of the blackbody radiation at the frequency of the observations) are 0.6 at a frequency $v_{1}=380 \mathrm{GHz}(\lambda=709 \mu \mathrm{m})$ and 3.4 at a frequency $v_{2}=624 \mathrm{GHz}(\lambda=481 \mu \mathrm{m})$.

As a possible mechanism for the formation of this distortion Lacey and Field (1988) considered Compton scattering of CBR photons by a hot intergalactic medium heated by powerful supernova explosions in earlier cosmological epochs. Another possibility for the interpretation of the spectral distortions of the CBR was proposed by Daly (1988) and Lacey and Field (1988), who examined emission of heated dust grains homogeneously distributed in the Universe in epochs with redshifts $z>5$. But this suggestion requires special mechanisms of dust heating at high redshifts.

In this paper we consider the possibility that this excess is the result of the total emission of galaxies in all cosmological epochs.

At the present time there is substantial observational data (absorption spectra of quasars, direct observations of $L_{\alpha}$-emission, etc.) which show that galaxies exist at $z \cong 3-4$. We define GMS (the number of galaxies with mass $\mathrm{M}$ per $\mathrm{Mpc}^{3}$ in an epoch with redshift $z$ ) in different cosmological epochs by the coagulation equation. The core of this equation is the rate coefficient of the coalescence. This coefficient may be found using the following assumptions: (i) Coalescence of two gravitationally interacting galaxies must occur if the lowest distance between them during their approach is less than or equal to the sum of the galaxy radii; (ii) At the moment of the closest approach, a decrease of the orbital energy must be about equal to this energy.

Approximate solutions of the coagulation equation were obtained for a pure exponential initial GMS at high redshifts. The parameters of the initial spectrum were selected in such way that in the modern epoch $(z=0)$ the solutions give the mass spectrum, which can be obtained from the observed spectrum of luminosities. We show our results in Figure 1. The mass spectrum at $z=0$ is shown by the dotted line. In the initial GMS we obtained the exponential parameter $\mathrm{M} \cong 10^{7} \mathrm{M}_{\odot}$. This means that the initial spectrum mainly consists of globular clusters $10^{6}-10^{7} \mathrm{M}_{\odot}$ at the epochs with redshifts $z_{0}$ which we consider as initial in our model.

If we know the ratio of the dust mass to the total mass of a galaxy, we can calculate the infrared flux for this galaxy. We suggest that the optical properties of the dust grains are similar to those in our Galaxy. It is necessary also to know the dust temperature. We consider two types of galaxies: normal galaxies and interacting galaxies for which star formation 


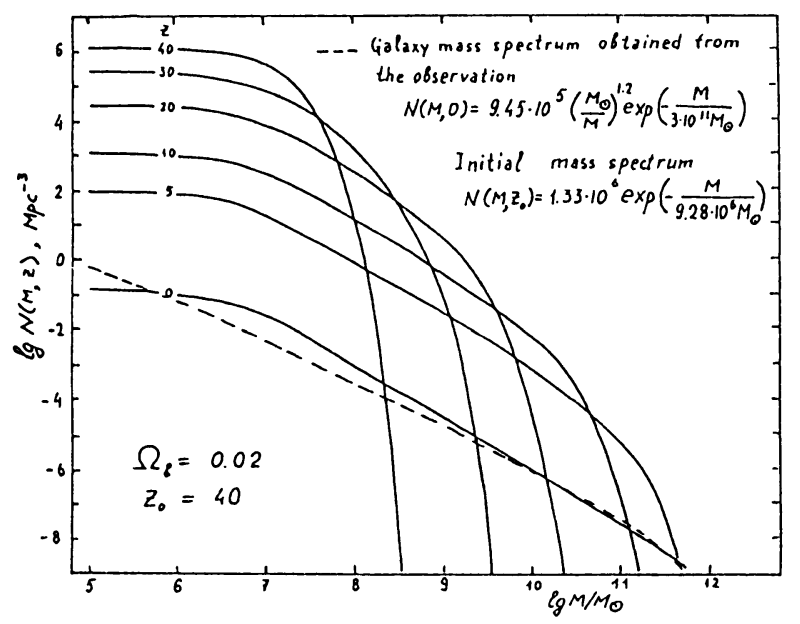

Figure 1. The evolution of the Galaxy Mass Spectrum during the Universe expansion from the initial GMS, N(M, $\left.z_{0}\right)$ to the modern GMS, $\mathrm{N}(\mathrm{M}, 0)$. $\mathrm{H}_{0}=55 \mathrm{~km} \mathrm{~s}^{-1}$ $\mathrm{Mpc}^{-1}, \Omega=1, \Omega_{b}=0.04$.

takes place on a scale of the whole galaxy. The number of galaxies of each type depends on the mass and redshift. Further, in these two types of galaxies the dust temperatures have different values $\mathrm{T}_{d}$ and $\mathrm{T}_{d}^{*}$ in accordance with the observational data concerning the infrared radiation of isolated and interacting galaxies in the modern epoch $(z=0)$.

To obtain the expected distortions of the CBR spectrum, it is necessary to sum the infrared radiation from galaxies of all masses in various cosmological epochs $0<z<z_{0}$, taking into account the wavelength redshift and the influence of the Universe curvature on the angular sizes of the sources.

The calculations were performed for the parameters $H_{0}=55 \mathrm{~km} \mathrm{~s}^{-1} \mathrm{Mpc}^{-1}, \Omega=1, \Omega_{b}=$ $0.02,0.04$ and $0.1, M_{\text {dust }} / M=0.003$. These calculations show that the absolute value of $\zeta\left(v_{2}\right)$ $=3.4$ can easily be obtained if, for example, $\Omega_{b}=0.04$ and $\mathrm{T}_{d}=40 \mathrm{~K}$ and $\mathrm{T}_{d}^{*}=65 \mathrm{~K}$. In this case $\zeta\left(v_{1}\right)=0.3$, which is less than the observed value. In order to obtain agreement between the calculated and observed contrast at two frequencies, it is necessary to determine the $z$-dependence of the dust temperature, which has a maximum at $z \cong 5$. The results obtained in this way are $\zeta\left(v_{1}\right) \cong 0.5$ and $\zeta\left(v_{2}\right) \cong 3.8$, which is close to the observed values. The corresponding intensities are shown in Figure 2 by the crosses.

\section{REFERENCES}

Daly, R. A. 1988, Astrophys. J., 333, L1.

Lacey, C. F., and Field, G. B. 1988, Astrophys. J., 330, $\mathrm{L} 1$.

Matsumoto, T., Mayakama, S., Matsuo, H., Marakami, H., Sato, S., Lange, A. E., and Richards, P. L. 1988, Astrophys. J., 389, 567.

Figure 2. The observed $(\bullet)$ and calculated $(+)$ excess of the background radiation in submillimeter wavelength region.

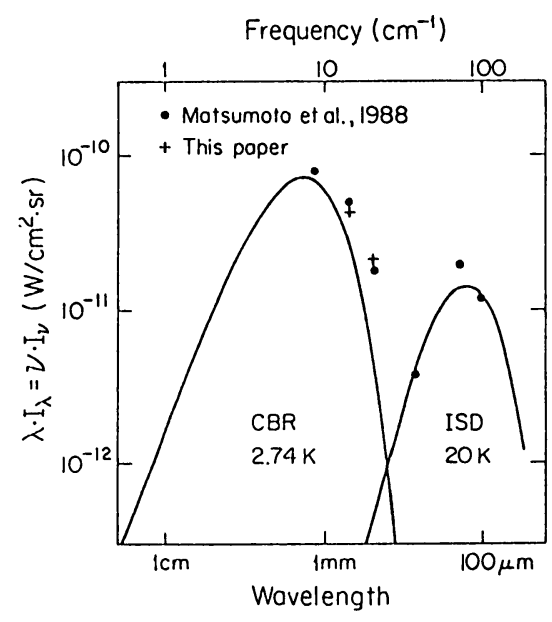

